\title{
A Global Registration Algorithm of the Single-Closed Ring Multi-Stations Point Cloud
}

\author{
Ronghua Yang ${ }^{1,2,3,{ }^{*}}$, Leixilan Pan ${ }^{1,2}$, Zejun Xiang ${ }^{3}$, Huaien Zeng ${ }^{4,5}$ \\ ${ }^{1}$ School of Civil Engineering, Chongqing University, Chongqing, 400045, China; \\ rh_y ang@cqu.edu.cn (R.Y.); 20151602005t@ @qu.edu.cn (L.P.); \\ ${ }^{2}$ Key Laboratory of New Technology for Construction of Cities in Mountain Area (Chongqing University), Ministry of Education, \\ Chongqing, 400045, China; \\ ${ }^{3}$ Chongqing Survey Institute, Chongqing, 401121, China; xiangzj@ cqkcy.com (Z.X.); \\ ${ }^{4}$ Hubei Key Laboratory of Intelligent Vision Based Monitoring for Hydroelectric Engineering, China Three Gorges University, \\ Yichang, 443002, China; zenghuaien_2003@163.com (H.Z.) \\ ${ }^{5}$ Key Laboratory of Geological Hazards on Three Gorges Reservoir Area (China Three Gorges University), Ministry of Education, \\ Yichang, 443002, China
}

Commission VI, WG VI/4

Keywords Terrestrial laser scanning; Multi-stations registration, ICP, Global registration, Single-Closed Ring, Point Cloud

\begin{abstract}
Aimed at the global registration problem of the single-closed ring multi-stations point cloud, a formula in order to calculate the error of rotation matrix was constructed according to the definition of error. The global registration algorithm of multi-station point cloud was derived to minimize the error of rotation matrix. And fast-computing formulas of transformation matrix with whose implementation steps and simulation experiment scheme was given. Compared three different processing schemes of multi-station point cloud, the experimental results showed that the effectiveness of the new global registration method was verified, and it could effectively complete the global registration of point cloud.
\end{abstract}

\section{INTRODUCTION}

In order to obtain the complete information of the object surface, multi-stations scanning of different perspectives is needed (Sharp G.C., Lee S.W., Wehe D.K., 2004). The obtained data from each station generally has a relatively independent coordinate system, which must be converted to the same coordinate system through a suitable multi-stations registration model. Therefore, how to accurately register the multi-stations point cloud was always a hot issue in point cloud data processing.

The registration of multi-stations point cloud is divided into the registration with target and without target., where the study of the latter was earlier than the former. Yang and Medioni (Yang C., Medioni G., 1992) first proposed the problem of multi-stations point cloud registration, and proposed that "the selected two point cloud were registered firstly, the other point cloud were registered to them in order", but it required that the selected two point cloud overlapped with all the other point cloud; Subsequently, Gagnon et al (Gagnon H., Soucy M., Bergevin R., Laurendeau D., 1994) proposed that "the firststation point clouds was registered with the other point cloud, then the second-station was registered with the other point cloud, and so on", however, the algorithm did not always converge, it was easy to fall into a dead cycle, and the iteration process was long and the computation was large; Bergevin et al (Bergevin R., Soucy M., Gagnon H., 1996) proposed that "the point cloud which overlapped the most other point cloud was selected as the reference point cloud, and the other were registered to it in order", but this method could not improve the registration accuracy of two stations with small overlap; Pulli (Pulli K., 1999) proposed that "For the single-closed ring multistations point cloud, the fore-and-aft point cloud were registered firstly, and then the other were registered to it in turn", but the registration accuracy of the middle point cloud was the worst; Sharp et al (Sharp G.C., Lee S.W., Wehe D.K., 2004) deduced the multi-stations cloud registration model under the constraint which was the minimum square sum of the angular error of the rotation matrix, which was the most theoretical multi-stations cloud registration algorithm, but the error of the rotation matrix was not equal to the angular error of the rotation matrix, and the calculation formulas of the correction matrix were still more complex; Guan (Guan Y., 2008) proposed that "the registration error of the last-station point cloud were corrected by the method of quadratic surface fitting", but the other point cloud registration error was ignored. In 2014, Yang R.H et al (Yang R.H., Lv M.Y., Hua X.H., 2014) proposed a new the global registration method of the multistations point cloud with target, showing the effectiveness of the method. However, the method of non-target has not yet been put forward.

In this paper, we focused on the global registration problem of the single-closed ring multi-stations point cloud, and proposed a new constraint condition which was different from 
Sharp et al (Sharp G.C., Lee S.W., Wehe D.K., 2004). Then, we deduced the correction model of the translation matrix, derived their fast-computing formula, and verified them by two examples.

\section{REGIS TRATION MODEL OF POINT CLOUD}

\subsection{Registration model of two-stations point cloud}

The transformation model of any two pieces of point cloud could be written as

$$
p^{i+1}=R_{i} p^{i}+T_{i}
$$

$R_{i}=\left[\begin{array}{lll}q_{0 i}^{2}+q_{1 i}^{2}-q_{2 i}^{2}-q_{3 i}^{2} & 2\left(q_{0 i} q_{3 i}+q_{1 i} q_{2 i}\right) & 2\left(q_{1 i} q_{3 i}-q_{0 i} q_{2 i}\right) \\ 2\left(q_{1 i} q_{2 i}-q_{0 i} q_{3 i}\right) & q_{0 i}^{2}-q_{1 i}^{2}+q_{2 i}^{2}-q_{3 i}^{2} & 2\left(q_{0 i} q_{1 i}+q_{2 i} q_{3 i}\right) \\ 2\left(q_{0 i} q_{2 i}+q_{1 i} q_{3 i}\right) & 2\left(q_{2 i} q_{3 i}-q_{0 i} q_{1 i}\right) & q_{0 i}^{2}-q_{1 i}^{2}-q_{2 i}^{2}+q_{3 i}^{2}\end{array}\right]$

$$
T_{i}=\left[\begin{array}{c}
t_{x i} \\
t_{y i} \\
t_{z i}
\end{array}\right], \quad q_{i}=\left[\begin{array}{c}
q_{0 i} \\
q_{1 i} \\
q_{2 i} \\
q_{3 i}
\end{array}\right]=\left[\begin{array}{c}
\cos \frac{\delta_{i}}{2} \\
\sin \frac{\delta_{i}}{2} \cdot \vec{n}_{i}
\end{array}\right], \quad \vec{n}_{i}=\left[\begin{array}{c}
n_{x i} \\
n_{y i} \\
n_{z i}
\end{array}\right],
$$

where $p^{i}=\left[x^{i}, y^{i}, z^{i}\right]^{T}$ and $p^{i+1}=\left[x^{i+1}, y^{i+1}, z^{i+1}\right]^{T}$ were the coordinates of any point in Scan $i$ and Scan $i+1 . R_{i}$ and $T_{i}$ were the rotation matrix and the translation vector. $\vec{n}_{i}$ and $\delta_{i}$ were the rotation axis and rotation angle of $R_{i}$ $\left(\angle R_{i}=\delta_{i}\right)$ and $\delta_{i} \in[-\pi, \pi]$ (Sharp, 2004)).

\subsection{Registration Model of Single Cycle Point Cloud}

The registration model of a single-closed ring multi-stations point cloud (Fig. 1) could be written as

$$
P_{1}=R_{n}\left(R_{n-1}\left(\cdots\left(R_{2}\left(R_{1} P_{1}+T_{1}\right)+T_{2}\right) \cdots\right)+T_{n-1}\right)+T_{n}
$$

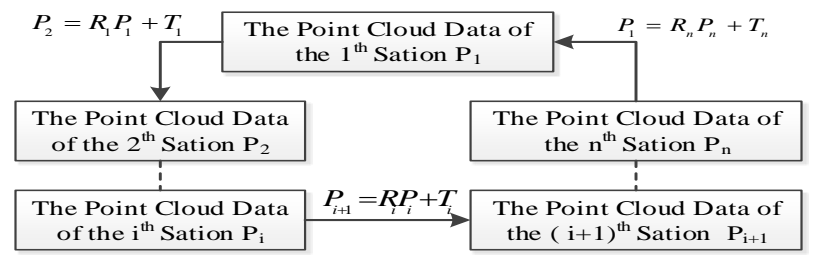

Fig.1 Regitration schematic graph of single-closed ring multi-stations poin cloud

The constraint conditions of Eq.4 were (Sharp, 2004)

$$
\left\{\begin{array}{l}
R_{n} \cdots R_{2} R_{1}=E \\
R_{n} \cdots R_{2} T_{1}+R_{n} \cdots R_{3} T_{2}+\cdots+R_{n} T_{n-1}+T_{n}=0
\end{array}\right.
$$

Using the above equation, we could get the correct model of the rotation matrix were (Sharp, 2004)

$$
\left\{\begin{array}{l}
\hat{R}_{1}=R_{1} B_{1} \\
\hat{R}_{2}=R_{2} R_{1} B_{2}\left(R_{1}\right)^{-1} \\
\cdots \\
\hat{R}_{n}=R_{n} \cdots R_{1} B_{n}\left(R_{n-1} \cdots R_{1}\right)^{-1}
\end{array}\right.
$$

where $\hat{R}_{n} \cdots \hat{R}_{2} \hat{R}_{1}=E, \hat{R}_{j}$ and $B_{j}$ were the modified matrix and correction matrix of $R_{j}$ respectively, $B_{j}$ was also a rotation matrix, and $j=1, \cdots, n$.

$$
\begin{gathered}
\text { Let } B_{n} \cdots B_{2} B_{1}=B, \quad \angle B=\beta, \\
\angle B_{j}=\alpha_{j} \in\left[-\frac{\pi}{2}, \frac{\pi}{2}\right] \text {, with Eq. 6, we could get (Sharp, }
\end{gathered}
$$
2004)

$$
\left\{\begin{array}{l}
B=\left[\begin{array}{lll}
b_{11} & b_{12} & b_{13} \\
b_{21} & b_{22} & b_{23} \\
b_{31} & b_{32} & b_{33}
\end{array}\right]=B_{n} \cdots B_{2} B_{1}=\left(R_{n} \cdots R_{2} R_{1}\right)^{T} \\
\alpha_{1}+\alpha_{2}+\cdots+\alpha_{n} \geq \beta
\end{array}\right.
$$

where the equality was also achieved if and only if $B_{1}, \ldots$, $B_{n}$ and $B$ had the same rotation axis.

With Eq. 5 and Eq. 6, we could get

$$
A t_{\Delta}=-A T_{0}
$$

where

$$
\begin{aligned}
& A=\left[\begin{array}{llllll}
\hat{R}_{n} \hat{R}_{n-1} \cdots \hat{R}_{2} & \hat{R}_{n} \hat{R}_{n-1} \cdots \hat{R}_{3} & \cdots & \hat{R}_{n} & I
\end{array}\right] \\
& T=\left[\begin{array}{c}
T_{1} \\
T_{2} \\
\vdots \\
T_{n}
\end{array}\right]=T_{0}+t_{\Delta} \quad, \quad T_{0}=\left[\begin{array}{c}
T_{10} \\
T_{20} \\
\vdots \\
T_{n 0}
\end{array}\right], \quad t_{\Delta}=\left[\begin{array}{c}
d t_{1} \\
d t_{2} \\
\vdots \\
d t_{n}
\end{array}\right]
\end{aligned}
$$

\section{GLOBAL REGISTRATION OF MULTI-STATIONS POINT CLOUD}

\subsection{Correction matrix of rotation matrix}

G.C. Sharp assumed the constraint condition was $\sum_{j=1}^{n} \alpha_{j}^{2}=\min \quad$ (Sharp, 2004). Here, we assumed the constraint condition was

$$
\sum_{j=1}^{n} \Delta_{R_{j}}^{2}=\min
$$




$$
\Delta_{R_{j}}=\hat{R}_{j}-R_{j}=R_{j}\left(R_{j-1} \cdots R_{1}\right) B_{j}\left(R_{j-1} \cdots R_{1}\right)^{T}-R_{j}
$$

With the Eq. 2, Eq. 3, Eq. 7 and Eq. 12, we could get

$$
\operatorname{tr}\left(\Delta_{R_{j}}^{T} \Delta_{R_{j}}\right)=6-2 \operatorname{tr}\left(B_{j}\right)=8 \sin ^{2} \frac{\alpha_{j}}{2}
$$

With the above Eq.13, the Eq.11 could be written as

$F=\sum 8 \sin ^{2}\left(\alpha_{j} / 2\right)+\lambda_{R}\left(\sum \alpha_{j}-c\right)=\min$

where $\sum_{j=1}^{n} \alpha_{j}=c, \lambda_{R}$ was a Lagrange coefficient.

Using Lagrange Adjustment Principle and Eq. 7, Eq. 13, Eq. 14 , we could get

$$
\left\{\begin{array}{l}
\lambda_{R}=-4 \sin \left(\frac{\beta}{n}\right) \\
\alpha_{1}=\cdots=\alpha_{n}=\frac{\beta}{n} \\
B_{1}=B_{2}=\cdots=B_{n}=B^{\frac{1}{n}}
\end{array}\right.
$$

The above model was consisted with the results in the paper of Sharp et al (Sharp G.C., Lee S.W., Wehe D.K., 2004).

\subsection{Fast-computing formula of correction matrix of rotation matrix}

With Eq. 2, Eq. 3 and Eq. 7, we could get

$$
B=\cos \beta \cdot I+\sin \beta \cdot M_{1}+(1-\cos \beta) M_{2}
$$

where

$$
\begin{gathered}
M_{1}=\frac{1}{2 \sin \beta}\left[\begin{array}{ccc}
0 & b_{12}-b_{21} & b_{13}-b_{31} \\
b_{21}-b_{12} & 0 & b_{23}-b_{32} \\
b_{31}-b_{13} & b_{32}-b_{23} & 0
\end{array}\right] \\
M_{2}=\frac{1}{2(1-\cos \beta)}\left[\begin{array}{ccc}
-b_{11}-b_{22}+b_{33}+1 & b_{12}+b_{21} & b_{13}+b_{31} \\
b_{12}+b_{21} & -b_{11}+b_{22}-b_{33}+1 & b_{23}+b_{32} \\
b_{13}+b_{31} & b_{23}+b_{32} & b_{11}-b_{22}-b_{33}+1
\end{array}\right] \\
\beta=\arccos \left(\frac{\operatorname{tr}(B)-1}{2}\right)
\end{gathered}
$$

With Eq.15 Eq.18, we could get

$B_{1}=B_{2}=\cdots=B_{n}=\cos \frac{\beta}{n} \cdot I+\sin \frac{\beta}{n} \cdot M_{1}+\left(1-\cos \frac{\beta}{n}\right) M_{2}$

The equation Eq. 6 and Eq. $16 \sim$ Eq. 20 were the fastcomputing formula of rotation matrix correction model.

\subsection{Fast-computing formula of correction matrix of translation vector}

With the above Eq. 5, we could get

$$
G=t_{\Delta}^{T} t_{\Delta}+\lambda_{T} A\left(T_{0}+t_{\Delta}\right)=\min
$$

where $\lambda_{T}$ was Lagrange coefficient, $A$ and $T_{0}$ were calculated by Eq.9 and Eq. 10, respectively.

Using Lagrange Adjustment Principle, we could get

$$
\left\{\begin{array}{l}
\lambda_{T}=-\frac{2}{n}\left(A T_{0}\right)^{T} \\
t_{\Delta}=\frac{1}{n} A^{T} A T_{0}
\end{array}\right.
$$

With Eq. 9, Eq. 10 and Eq. 22, we could get the fast-computing formula of translation matrix correction model

$$
\left\{\begin{array}{l}
d t_{n}=\frac{1}{n} A T_{0} \\
d t_{n-1}=\hat{R}_{n}^{T} d t_{n} \\
d t_{n-2}=\left(\hat{R}_{n-1}\right)^{T} d t_{n-1} \\
\cdots \\
d t_{1}=\left(\hat{R}_{2}\right)^{T} d t_{2}
\end{array}\right.
$$

\subsection{Algorithm implementation step}

Based on the previous discussion, the following procedure may be adopted for the single-closed ring multi-station point cloud:

Step 1 Let $k=0$, where $k$ denotes iterative number, and its threshold was $m$;

Step 2 Let $\tau=0.1$, where $\tau$ denotes the angular closure error threshold-value of the single-closed ring multi-station point cloud;

Step 3 Register any two-adjacent point cloud by ICP algorithm, and get the initial-value of the transformation matrices $\left\{\left(R_{j}, T_{j}\right)\right\}, j=1, \cdots, n$;

Step 4 Calculate the rotation angle $\beta$ of $B$ by Eq. 7 and Eq. 19 ;

Step 5 If $k>m$, go to Step 13; if not, $k=k+1$ and continue;

Step 6 If $\beta>\tau$, go to Step 13; if not, continue;

Step 7 Calculate the rotation correction matrix $B_{j}$ by Eq. 17, Eq. 18 and Eq. 20;

Step 8 Calculate the modified matrices $\left\{\hat{R}_{j}\right\}$ by Eq. 6; 
Step 9 Calculate the correction matrices $\left\{d t_{i}\right\}$ by Eq. 9 , Eq. 10 and Eq. 23;

Step 10 Calculate the modified matrices $\left\{\hat{T}_{j}\right\}$ by Eq. 23;

Step 11 Register all point cloud, go back to Step 3;

Step 12 Output the registered point cloud.

\section{EXPERIMENT ANALYS IS}

\subsection{Registration $S$ chemes}

In order to analysis the effectiveness of the proposed global registration algorithm, we designed three kinds of processing schemes:

Scheme A: no correction, corresponding the step 3 and step 12 in the above section 3.4;

Scheme B: no iteration, corresponding the step $1 \sim$ step 12 and $m=1$ in the above section 3.4;

Scheme C: iteration and correction, corresponding the step $1 \sim$ step 12 and $m \neq 1$ in the above section 3.4.

\subsection{Data simulation}

The registration accuracy of ICP was related to the measurement accuracy of point, the searching accuracy of the point-correspondences and so on. So we simulated three types of point cloud as follows:

Step 1 Construct a single-closed ring 4-stations point cloud $\tilde{D}_{\text {first } 1}, \quad \tilde{D}_{\text {first } 2}, \quad \tilde{D}_{\text {first } 3}, \quad \tilde{D}_{\text {first } 4}$ (denoted as $\left\{\tilde{D}_{\text {first } 1 \sim 4}\right\}$ ) by the point cloud of a cube (each face contains five points), where each point cloud included ten points in two adjacent surface of the cube (see Fig. 2(a));

Step 2 Simulate randomly 500 transformation parameters $\left\{R_{2}^{i}, R_{3}^{i}, R_{4}^{i}, T_{2}^{i}, T_{3}^{i}, T_{4}^{i}\right\}, i=1, \cdots, 500$;

Step 3 Transform the point cloud $\left\{\tilde{D}_{\text {first } 1 \sim 4}\right\}$ by the above transformation parameters and Eq. 1, and add the Gaussian white noise (with a $2.5 \mathrm{~mm}$ variance) to the transferred point cloud, we obtained the point cloud $\left\{D_{\text {first } 1 \sim 4}^{i}\right\}$, $i=1, \cdots, 500$ (denoted as Group I);

Step 4 Obtain the Bunny point cloud from the Stanford Computer Graphics Laboratory website (see Fig. 2(b));

Step 5 Construct a single-closed ring 7-stations point cloud $\tilde{D}_{\text {second } 1}, \cdots, \tilde{D}_{\text {second } 7}$ (denoted as $\left.\left\{\tilde{D}_{\text {second } 1 \sim 7}\right\}\right)$ by counter-clockwise extraction from the Bunny point cloud, where any adjacent two point cloud had overlap over 2/3;

Step 6 Simulate randomly 500 transformation parameters $\left\{R_{2}^{i}, \cdots, R_{7}^{i}, T_{2}^{i}, \cdots, T_{7}^{i}\right\}, i=1, \cdots, 500$;
Step 7 Transform the point cloud $\left\{\tilde{D}_{\text {second } 1 ~ 7}\right\}$ by the above transformation parameters and Eq. 1, we obtained the point cloud $\left\{D_{\text {second 1 7 }}^{i}\right\}$ (denoted as Group II);

Step 8 Add the Gaussian white noise (with a $2.5 \mathrm{~mm}$ variance) to the point cloud $\left\{D_{\text {second1 7 }}^{i}\right\}$, we obtained the point cloud $\left\{D_{\text {third 1 7 }}^{i}\right\}$ (denoted as Group III).

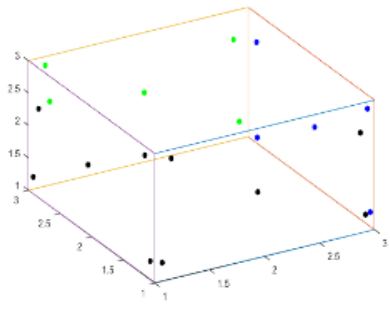

Fig. 2(a)

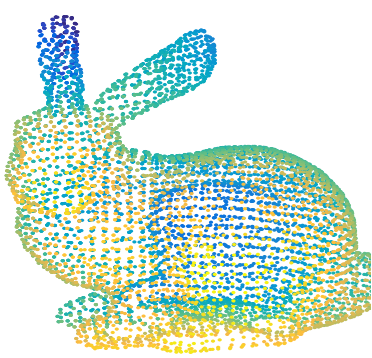

Fig. 2(b)
Fig 2. The raw data

\subsection{Registration processing}

The registration accuracy of ICP was related to the measurement accuracy of point, the searching accuracy of the point-correspondences and so on. So we simulated three types of point cloud as follows:

\subsubsection{Group I}

Step 1 Let $i=1$;

Step 2 Calculate the point cloud $\left\{D_{\text {first } 1 \sim 4}^{i}\right\}$ according to the scheme $\mathrm{A}, \mathrm{B}$ and $\mathrm{C}$ respectively, and the results were $\left\{\hat{D}_{\text {first } 1 \sim 4}^{i, A}\right\},\left\{\hat{D}_{\text {first } 1 \sim 4}^{i, B}\right\}$ and $\left\{\hat{D}_{\text {first } 1 \sim 4}^{i, C}\right\}$;

Step 3 Calculate the maximum-values and average-values of the registration error

$\left\{\begin{array}{l}\varepsilon_{\max , \text { first } 1 \sim 4}^{i, A}=\max \left(\left|\hat{D}_{\text {first } 1 \sim 4}^{i, A}-\tilde{D}_{\text {first } 1 \sim 4}\right|\right) \\ \varepsilon_{\max , \text { first } 1 \sim 4}^{i, B}=\max \left(\left|\hat{D}_{\text {first } 1 \sim 4}^{i, B}-\tilde{D}_{\text {first } 1 \sim 4}\right|\right) \\ \varepsilon_{\max , \text { first } 1 \sim 4}^{i, C}=\max \left(\left|\hat{D}_{\text {first } 1 \sim 4}^{i, C}-\tilde{D}_{\text {first } 1 \sim 4}\right|\right)\end{array}\right.$

$\left\{\begin{array}{l}\varepsilon_{\text {mean, first } 1 \sim 4}^{i, A}=\operatorname{mean}\left(\left|\hat{D}_{\text {first } 1 \sim 4}^{i, A}-\tilde{D}_{\text {first } 1 \sim 4}\right|\right) \\ \varepsilon_{\text {mean, first } 1 \sim 4}^{i, B}=\operatorname{mean}\left(\left|\hat{D}_{\text {first } 1 \sim 4}^{i, B}-\tilde{D}_{\text {first } 1 \sim 4}\right|\right) \\ \varepsilon_{\text {mean, first } 1 \sim 4}^{i, C}=\operatorname{mean}\left(\left|\hat{D}_{\text {first } 1 \sim 4}^{i, C}-\tilde{D}_{\text {first } 1 \sim 4}\right|\right)\end{array}\right.$

Step 4 If $i \leq 500$, go to Step 2; if not, continue; 
Step 5 Output the maximum-values, the average-value and the total mean-squared-error (see Fig. 3 and Fig. 4, where Scheme A was blue, Scheme $\boldsymbol{B}$ and $\boldsymbol{C}$ was red).

$$
\begin{gathered}
\left\{\varepsilon_{\text {max }, \text { first } 1 \sim 4}^{i, A}\right\},\left\{\varepsilon_{\text {max }, \text { first } 1 \sim 4}^{i, B}\right\},\left\{\varepsilon_{\text {max }, \text { first } 1 \sim 4}^{i, C}\right\} \\
\left\{\varepsilon_{\text {mean }, \text { first } 1 \sim 4}^{i, A}\right\},\left\{\varepsilon_{\text {mean }, \text { first } 1 \sim 4}^{i, B}\right\}, \\
\left\{\varepsilon_{\text {mean }, \text { first } 1 \sim 4}^{i, C}\right\}
\end{gathered}
$$

\section{Group II and Group III}

Step 1 Let $i=1$;

Step 2 Calculate $\left\{D_{\text {second } 1 \sim 7}^{i}\right\}$ and $\left\{D_{\text {third1 7 }}^{i}\right\}$ by the scheme A, B and C respectively;

Step 3 Calculate the maximum-values and average-values (similar to the above method in Group I);

Step 4 If $i \leq 500$, go to Step 2; if not, continue;

Step 5 Output the maximum-values and the average-values of Group II (see Fig. 5 and Fig. 6) and Group III (see Fig. 7 and Fig. 8), where Scheme $\boldsymbol{A}$ was blue, Scheme $\boldsymbol{B}$ was green and Scheme $C$ was red.

$$
\begin{gathered}
\left\{\varepsilon_{\max , \sec \text { ond } 1 \sim 7}^{i, A}\right\},\left\{\varepsilon_{\text {max,sec ond } 1 \sim 7}^{i, B}\right\}, \\
\left\{\varepsilon_{\text {max,sec ond } 1 \sim 7}^{i, C}\right\}
\end{gathered}
$$

$$
\begin{gathered}
\left\{\varepsilon_{\text {mean }, \text { sec ond } 1 \sim 7}^{i, A}\right\},\left\{\varepsilon_{\text {mean }, \text { sec ond } 1 \sim 7}^{i, B}\right\} \\
\left\{\varepsilon_{\text {mean }, \text { sec ond } 1 \sim 7}^{i, C}\right\} \\
\left\{\varepsilon_{\text {max }, \text { third } 1 \sim 7}^{i, A}\right\},\left\{\varepsilon_{\text {max }, \text { third } 1 \sim 7}^{i, B}\right\}, \\
\left\{\varepsilon_{\text {max }, \text { third } 1 \sim 7}^{i, C}\right\} \\
\left\{\varepsilon_{\text {mean,third } 1 \sim 7}^{i, A}\right\},\left\{\varepsilon_{\text {mean }, \text { third } 1 \sim 7}^{i, B}\right\} \\
\left\{\varepsilon_{\text {mean }, \text { third } 1 \sim 7}^{i, C}\right\}
\end{gathered}
$$

\subsection{Results analysis}

From Fig. $3 \sim$ Fig. 8 , it may be concluded that

(a) The maximum-values and average-values of processed results by scheme $\mathrm{C}$ were better than by scheme $\mathrm{B}$, which mean that the more iterations, the better results;

(b) The maximum-values and average-values of processing results by scheme B were better than by scheme A, which mean that the proposed global registration model could improve the accuracy of ICP;

(c) The maximum-values of Group II were slightly smaller than the results of Group III, which mean that the noise had some effect on the registration accuracy.

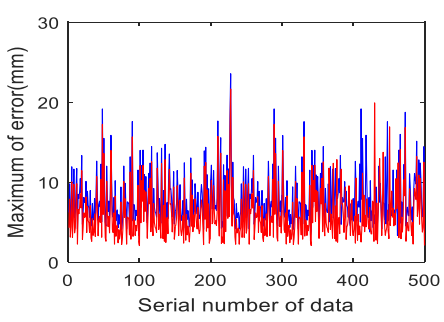

Fig. 3(a) Dfirst2

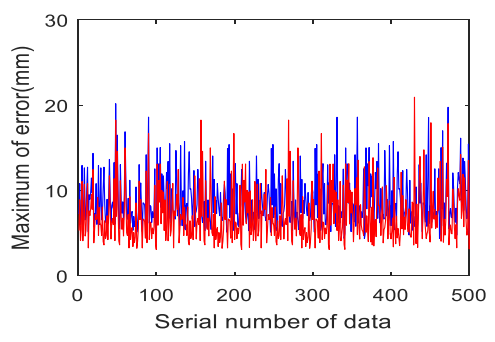

Fig. 3(b) Dfirst3

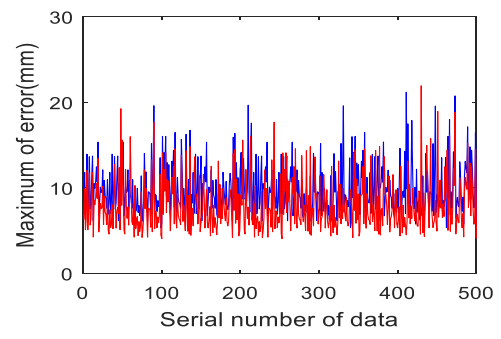

Fig. 3(c) Dfirst4

Fig 3 The maximum values of registration error (Group I)

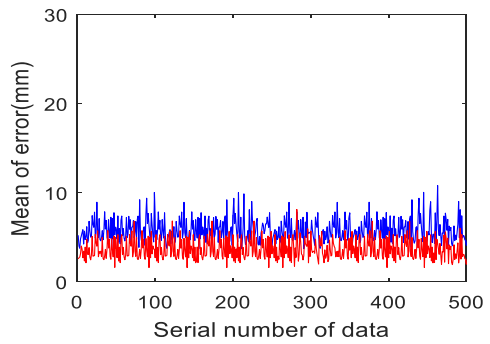

Fig. 4(a) Dfirst2

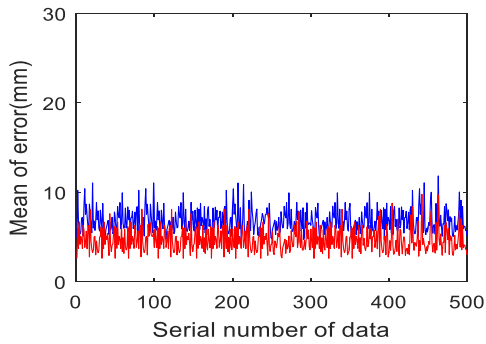

Fig. 4(b) Dfirst3

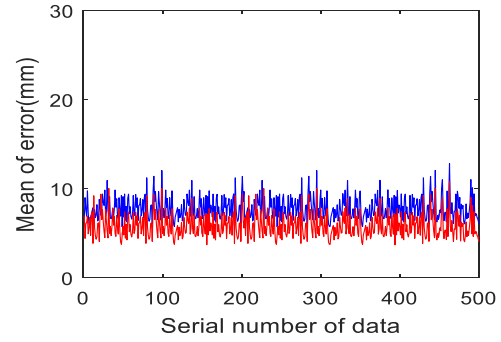

Fig. 4(c) Dfirst4 
The International Archives of the Photogrammetry, Remote Sensing and Spatial Information Sciences, Volume XLII-3, 2018 ISPRS TC III Mid-term Symposium “Developments, Technologies and Applications in Remote Sensing”, 7-10 May, Beijing, China

Fig 4 The mean- values of registration error (Group I)

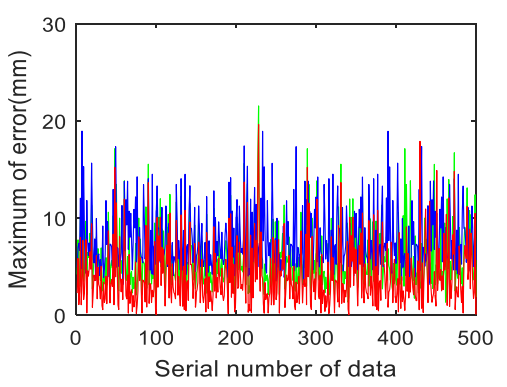

Fig. 5(a) Dsecond2

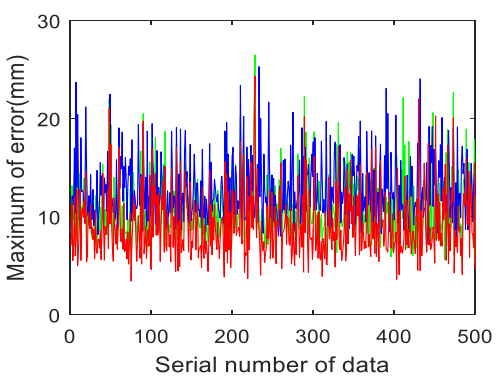

Fig. 5(d) Dsecond5

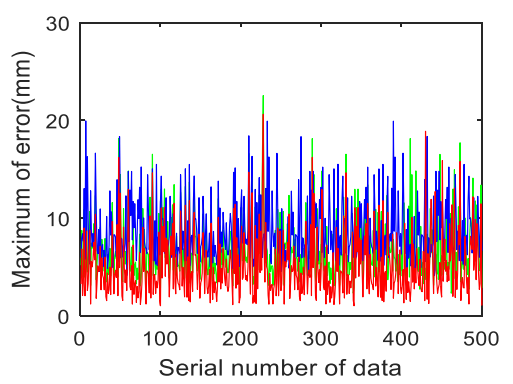

Fig. 5(b) Dsecond3

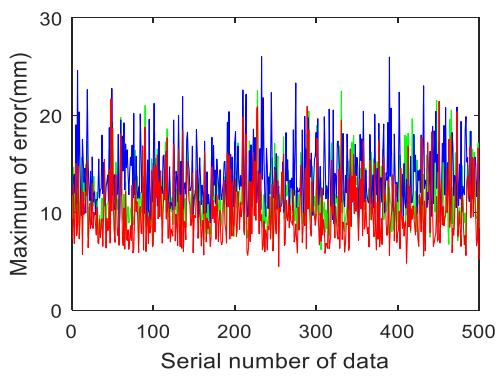

Fig. 5(e) Dsecond6

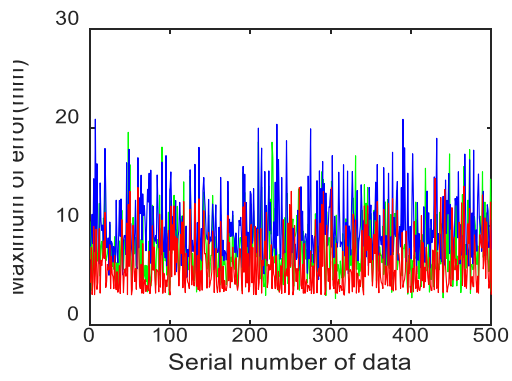

Fig. 5(c) Dsecond4

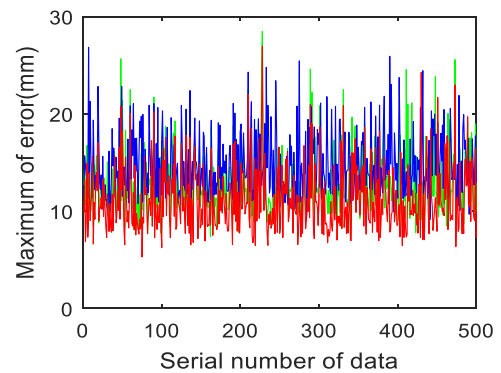

Fig. 5(f) Dsecond7

Fig. 5 The maximum-values of registration error (Group II)

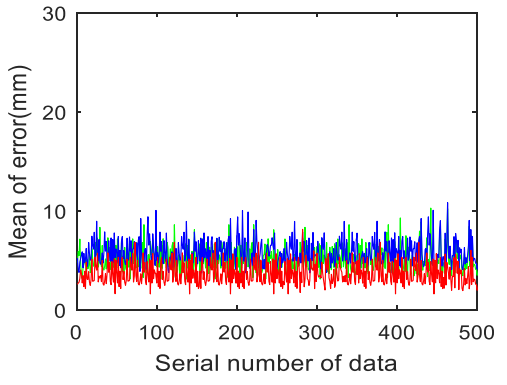

Fig. 6(a) Dsecond2

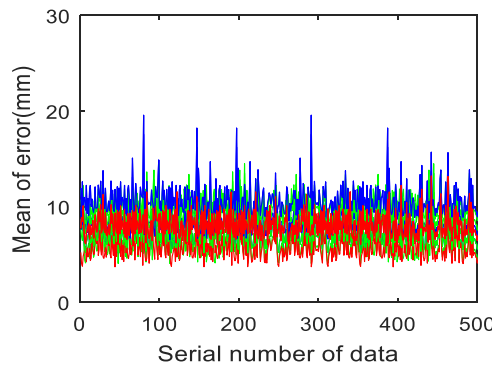

Fig. 6(d) Dsecond5

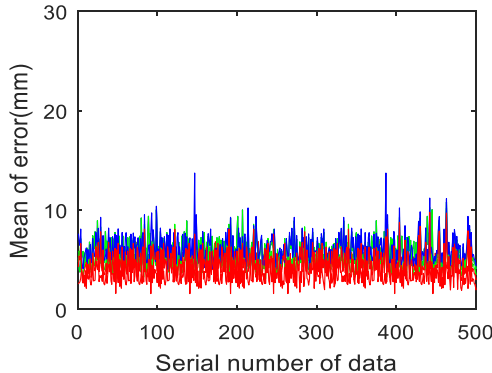

Fig. 6(b) Dsecond3

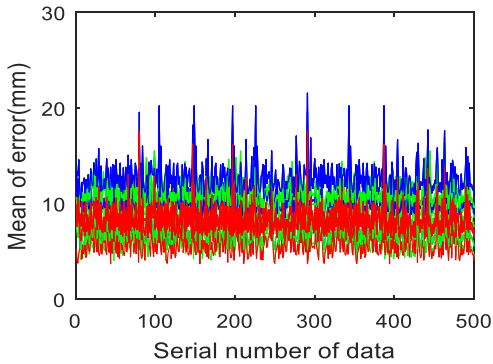

Fig. 6(e) Dsecond6

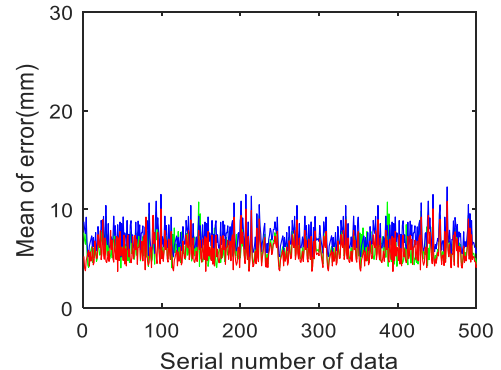

Fig. 6(c) Dsecond4

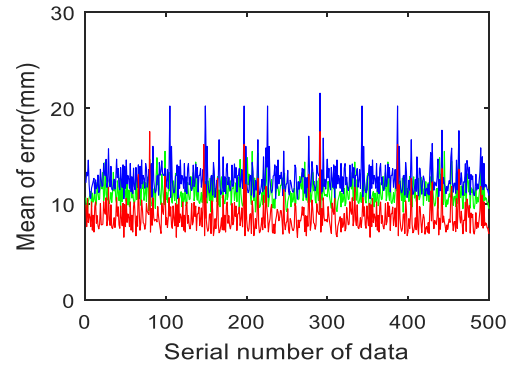

Fig. 6(f) Dsecond7

Fig. 6 The mean-values of registration error (Group II) 


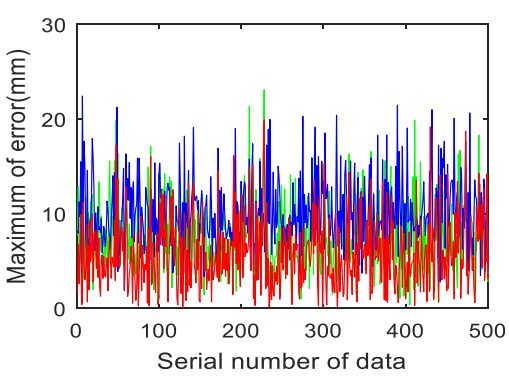

Fig. 7(a) Dthird2

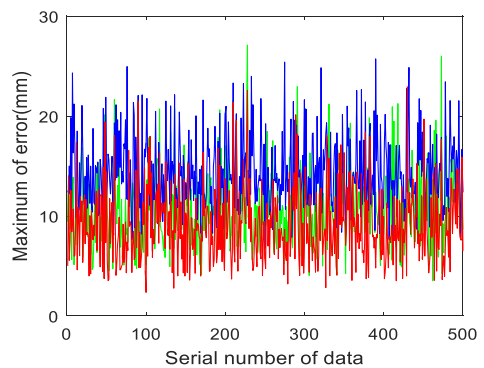

Fig. 7(d) Dthird5

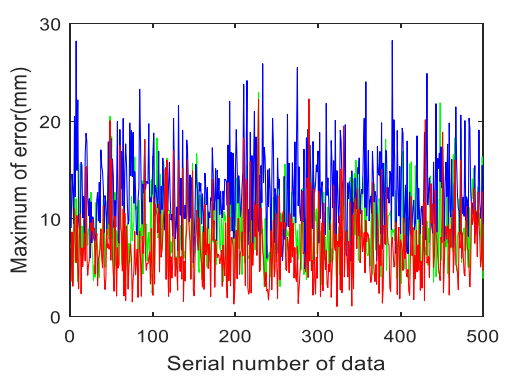

Fig. 7(b) Dthird3

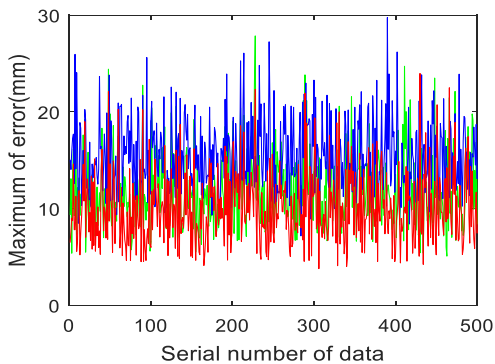

Fig. 7(e) Dthird6

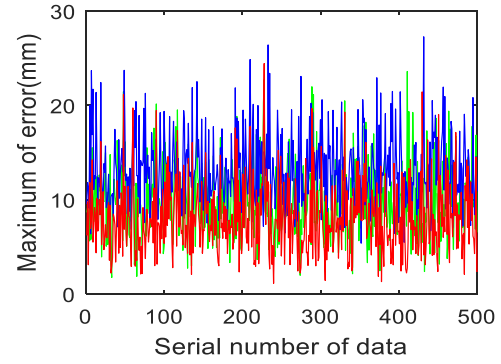

Fig. 7(c) Dthird4

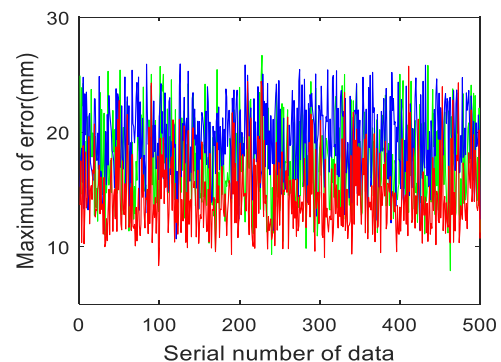

Fig. 7(f) Dthird7

Fig. 7 The maximum-values of registration error (Group III)

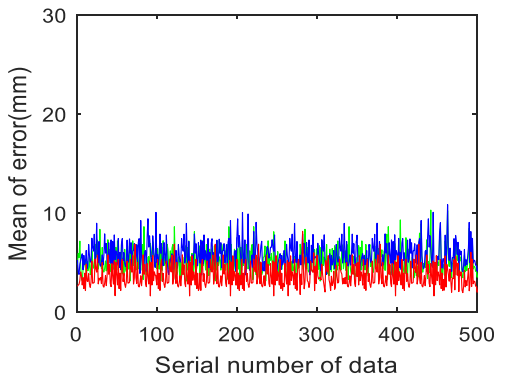

Fig. 8(a) Dthird2

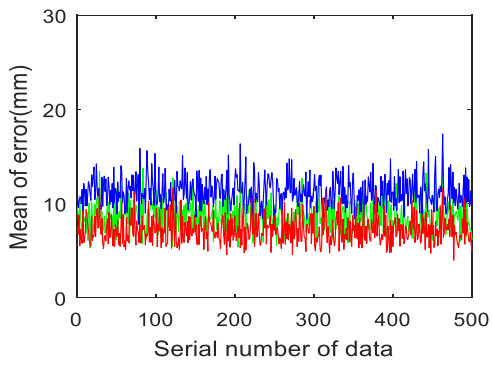

Fig. 8(d) Dthird5

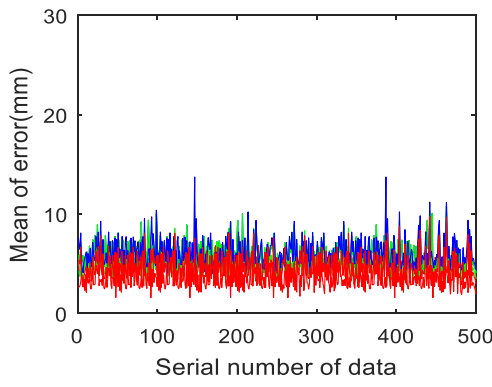

Fig. 8(b) Dthird3

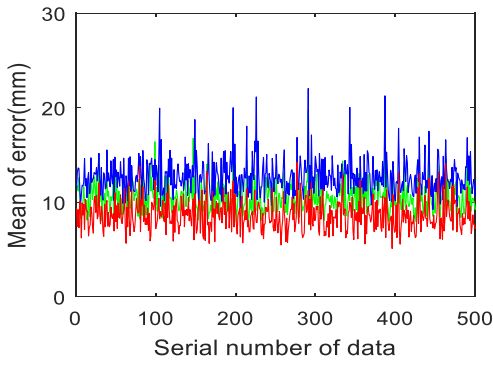

Fig. 8(e) Dthird6

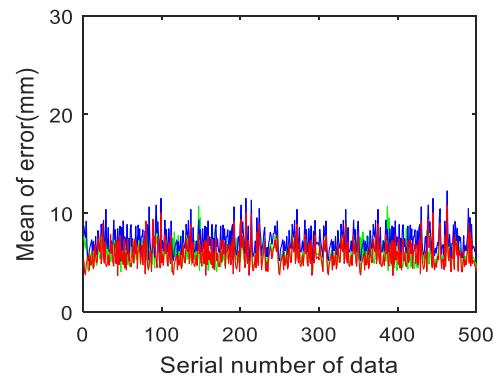

Fig. 8(c) Dthird4

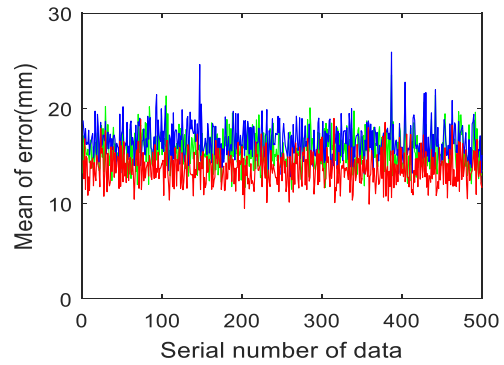

Fig. 8(f) Dthird7

Fig. 8 The mean-values of registration error (Group III)

\section{Conclusion}

A global registration algorithm of multi-stations point cloud had been a hot topic in the field of point cloud registration. In this paper, the correction model of rotation matrix was deduced under the new constraint condition that the square sum of the correction-values of the rotation matrix elements was minimized, and the fast-computing formulas of transformation matric were proposed. In addition, the new global registration algorithm was presented, and the effectiveness of three different processing 
schemes were compared. From the results of experiment, we could find that the global registration algorithm would improve

Acknowledgments This work was supported by the National Natural Science Foundation of China (No. 41304001), Chongqing Natural Science Foundation and Frontier Research Planning Project (No. cstc2014jcyjA00011), the Fundamental Research Funds for the Central Universities (No.0218005208005 and No.0903005203182), and the National Natural Science Foundation of Hubei Province (No. 2016CFB443).

Disclosure statement: No potential conflict of interest was reported by the authors.

\section{References}

Bergevin R., Soucy M., Gagnon H., Laurendeau D.. Towards a general multi-view registration technique. IEEE Transaction on Pattern Analysis and Machine Intelligence. 1996, pp.540-547.

Gagnon H., Soucy M., Bergevin R., Laurendeau D.. Registration of multiple range views for automatic 3-D model building. IEEE computer society conference on computer vision and pattern recognition. 1994, pp.581-586.

Guan Y.. Some problems of the processing of terrestrial laser scanning. PhD thesis in Tongji University. 2008.

Lu T., Zhou S., Zhang L. and Guan Y.. Sphere target fixing of point cloud based on TLS. Journal of Geodesy and Geodynamics. 2009, 29(4), pp.102-105.

Pulli K.. Multi-stations registration for large data sets, 2th International Conference on 3D Digital Imaging and modelling. 1999, pp.160-168. the registration accuracy.

Sharp G.C., Lee S.W., Wehe D.K.. Multi-stations registraion of 3D scenes by minimizing error between coordinate frames. IEEE Transactions on pattern analysis and machine intelligence. 2004, 26(8), pp.1037-1050.

Surveying Adjustment Team of School of Geodesy and Geomatics of Wuhan University. Error theory and foundation of surveying adjustment. Wuhan University Press. 2012, 2st ed., Wuhan, China.

Wouldiams J., Bennamoun M.. A multiple view 3D registration algorithm with statistical error modelling. IEICE Transaction on Information and Systems. 2000, pp.1662-1670.

Yang C., Medioni G.. Object modelling by registration of multiple range images. Image and Vision Computing. 1992, 10(3), pp.145-155.

Wang G.. Research on registration of ground lidar range images Master thesis in Beijing University of Civil Engineering and Architecture. 2006.

Yang R.. Research on point cloud angular resolution and processing model of terrestrial laser scanning. PhD thesis in Wuhan University. 2011.

Yang R.H., Lv M.Y., Hua X.H., An Alogrithm for the multoview target point cloud global registration. Geomatics and information science of Wuhan University. 2014 